\title{
Using Filtered and Semi-Continuous High Rate GPS for Monitoring Deformations
}

\author{
A.E.Ragheb ${ }^{1}$, S.J.Edwards ${ }^{2}$ and P.J.Clarke ${ }^{3}$
}

\begin{abstract}
Multipath is a major sidereally-repeating error affecting precise GPS positioning and deformation monitoring. Because satellite-receiver geometry repeats almost exactly every sidereal day, filtering can reduce multipath in near-static situations. Here, we investigate how sidereal filtering can be used in a switched multi-antenna array system providing semicontinuous GPS data, which may be adopted in order to reduce hardware costs.

Depending on the receiver's set-up, the optimum session switching interval is 119 seconds or a multiple thereof. This provides sufficient reliable epochs using a short switching interval, and is synchronized with the appropriate geometry repeat interval for sidereal filter application. The semi-continuous sidereally-filtered GPS technique is efficient in detecting horizontal and vertical displacements, surpassing $5 \mathrm{~mm}$ and $8 \mathrm{~mm}$ precision respectively per epoch in near-static environments with moderately high multipath. This provides the capability of monitoring deformations occurring at periods greater than double the switching interval, although it can also be used for monitoring rapid structural deformations despite data gaps which may affect temporal resolution. Consequently, this combined method presents an efficient, cost-effective and precise GPS technique.
\end{abstract}

Keywords: GPS, Sidereal Filter, Deformation Monitoring, Multi-Antenna Switch Array

${ }^{1}$ School of Civil Engineering and Geosciences, Newcastle University, Newcastle NE1 7RU, $U K$

Now: Assistant Professor, Public Works Department, Faculty of Engineering, Ain Shams University, Cairo, Egypt (aragheb@link.net)

${ }^{2}$ Senior Lecturer, School of Civil Engineering and Geosciences, Newcastle University, Newcastle NE1 7RU,UK (s.j.edwards@ncl.ac.uk)

${ }^{3}$ Professor, School of Civil Engineering and Geosciences, Newcastle University, Newcastle NE1 7RU, UK (peter.clarke@ncl.ac.uk)

An edited version of this paper was published by the American Society of Civil Engineers: 


\section{Introduction}

Monitoring of natural and structural deformations is a vital procedure with many benefits, such as assuring the performance of a structure against its design criteria, identifying abnormalities in the structure's behaviour, or early warning of geohazards. Also, it could be useful for calibration of structural design codes (Ogaja et al. 2001). Deformation monitoring is often required to be carried out as quickly as possible, that is in real time or near real time, for safety and stability purposes. This may help avoid the collapse of defective structures causing major catastrophes with ensuing financial liability or environmental damage (Rutledge et al. 2001).

GPS is an important tool for monitoring deformation, because it is reliable, all-weather, and capable of automation. However, as with all survey techniques it has drawbacks, such as the possibility of detecting false movements due to cycle slips or multipath, amongst other GPS error sources (Roberts et al. 2001, 2004). To achieve the required precision to monitor movements such as settlement of buildings, sometimes long or high rate continuous GPS sessions are required which lead to higher operational cost.

GPS can be applied to deformation monitoring using several observation strategies. The most precise is continuous static GPS, in which GPS receivers occupy all monitoring stations, while logging data continuously over long periods of time. Campaign GPS is also a popular technique, whereby data collection for an area is undertaken typically for a few hours, and repeated at long intervals e.g. yearly. Another case of static GPS is episodic GPS, where data is logged at each station for a limited time interval, and this is repeated after a short time gap, resulting in semi-continuous GPS data for each station. Kinematic GPS allows instantaneous GPS positioning at each station and at intervening locations, albeit with worse precision than the static case. Kinematic GPS also allows the monitoring of deformations occurring more rapidly, at periods from the Nyquist interval (twice the observation rate) through to typically minutes or hours.

Previous investigations such as Bock et al. (2000), De Jonge et al. (2000) and Bock et al. (2001) demonstrated epoch-by-epoch positioning as a viable alternative to traditional GPS batch processing, allowing a better insight into GPS error sources. Using this technique to accurately monitor deformations at high rates, many factors must be considered. The first is to use a rapid ambiguity resolution technique, e.g. single epoch, to allow reliable positioning even in the presence of cycle slips. 
Secondly, although most of the errors affecting short-baseline GPS are eliminated or minimized by differencing techniques (Leick 2004), multipath error will remain due to the highly site-specific nature of the reflection of GPS signals from nearby surfaces. Hence, multipath is often considered the most limiting factor in precise GPS positioning (e.g. Lau and Mok 1999; Axelrad et al. 1996). Multipath gives rise to errors with periods of a few seconds to minutes, but fortunately, due to the nearly exact repetition of satellite geometry relative to a site every sidereal day (nominally $23 \mathrm{~h} 56 \mathrm{~m} 04 \mathrm{~s}$ ), multipath and any other geometrically-repeating errors are highly correlated across subsequent days. This is, of course, valid providing the same antenna and reflector environment is maintained. Consequently, it is possible to apply "sidereal filtering" techniques to mitigate this error (Genrich and Bock 1992; Nikolaidis et al. 2001). Several recent investigations (Choi et al. 2004; Larson et al. 2007; Agnew and Larson 2007; Ragheb et al. 2007a) have shown that the actual satellite geometry repeat interval ("sidereal lag") is slightly less than the nominal sidereal period used in earlier sidereal filtering studies, and that it varies at the few-second level across the constellation.

Finally, one of the major drawbacks of continuous GPS is the high capital cost of the GPS equipment required at each site (Chen et al. 2000). Each installation will include a receiver, antenna, communications hardware and software, power supply (batteries or mains operated) and security fixtures. Thus, it is desirable to moderate this expense, which could be achieved using episodic GPS in the form of a switched multi-antenna array. This procedure has the advantage of combining some characteristics of both the static and rapid static techniques. GPS data are not collected continuously, but for a certain time interval at fixed antennae connected to one receiver, without the need to move a rover receiver through the monitoring points.

In this case, multiple GPS antennae are connected to a receiver which records data continuously whilst switching between antennae periodically (Ding et al. 2000). This will, of course, reduce the overall capital cost of the whole monitoring process significantly, due to the reduction in the number of receivers used compared with continuous GPS. Although the number of GPS antennae is unchanged, these have significantly lower unit costs. Each antenna is mounted at the required test location, and the signal from each antenna is multiplexed to the receiver through a switch mechanism (consisting of multiple input channels and a single output channel). This allows the receiver to sample sequentially the 
signals from each antenna for a certain time interval, producing periodic GPS data for the network of fixed monitoring stations (He et al. 2004a). Of course, for relative positioning, another receiver and antenna must be fixed at a nearby base station with known coordinates. Accordingly, GPS multi-antenna systems represent an economical and precise technique for deformation monitoring especially for high-density networks (He et al. 2005).

The main aim in our study is to use a fast, and more importantly accurate, GPS technique, with a single epoch processing approach (therefore without initialisation delay and cycle slip free), along with sidereal filtering (to minimize the most limiting factor of multipath error), and a switched antenna array procedure (to reduce cost), for the sake of monitoring structural deformations. In other words, the whole process is required to be carried out, a) quickly using a single epoch processing technique i.e. identifying the movement in real-time or near real-time in order to act immediately to reduce the impact of disasters, b) with minimum cost through the use of a dual antenna switch array, and c) precisely, by using the sidereal filter methodology.

The first investigation here is to determine the most appropriate observation session length per antenna before switching to the next one. We consider three main factors. The first is efficiency, that is using the shortest reliable session length that provides a useful quantity of data. The second is how to combine sidereal filtering with antenna switching, that is synchronizing the session length with the optimum sidereal lag. Thirdly, we aim to acquire the best possible precision for station coordinates.

Once we have established this objective, we demonstrate the utility and effectiveness of the technique, using a switched dual antenna array, collecting high rate $(1 \mathrm{~Hz})$ GPS data. The accuracy of deformation monitoring by this method is tested by simulating small $(1-100 \mathrm{~mm})$ antenna displacements using a calibrated moveable antenna platform.

\section{Single Epoch and Sidereally Filtered GPS}

The single epoch positioning approach used in this paper forms a search volume of possible trial positions using the double difference observables, seeking the correct ambiguity set at each epoch independently from any previous epochs. The algorithm is encoded in a software package called GPS Ambiguity Search Program (GASP) (Corbett 1994; Al-Haifi 1996; Gunasingam 2003; Ragheb 2008). As each epoch is treated separately, common parameters cannot affect the apparent level of multipath error. In addition it has the advantage of not 
requiring long GPS sessions or initialization periods, as well as eliminating the effect of cycle slips and the necessity for continuous lock on satellites. The program uses the ambiguity function technique (AFT) for resolving the phase integer ambiguities (Counselman and Gourevitch 1981; Remondi 1984). The principle behind this technique is to use complex number representation to separate the initial phase ambiguities and the fractional phases. Then, the ambiguity function value (AFV) is calculated for each trial position in the ambiguity search volume. Each candidate position having an AFV greater than a specified threshold is passed to the least squares adjustment procedure and the F-test statistic is used to select the final correct position and accompanying ambiguity set.

The use of sidereal filtering alongside single-epoch positioning was discussed by Ragheb et al. (2007a, 2007b), who found it to be efficient in mitigating multipath error, and thus improve the precision of the coordinates. Sidereal filtering is applied by removing from the point coordinates at each processed epoch the residual coordinates (difference from the longterm mean) at the corresponding epoch separated by the sidereal error repeat interval. Ragheb et al. (2007a, 2007b) confirmed that for double-difference processing the optimal lag to use in order to stack coordinate or phase residuals is 23h 55m 54s, i.e. 86154 s, that is 10 seconds earlier than the nominal geometry repeat interval, in agreement with previous investigations (e.g. Choi et al. 2004; Agnew and Larson 2007) using different methodologies. However, when double difference processing is adopted, using a lag that differs from the optimum by less than \pm 5 seconds has no appreciable effect on the final coordinate precision (Ragheb et al. 2007b).

Ragheb et al. (2007a) also discussed the ageing of any sidereal filter using different separations between the days when the residual coordinates were obtained and those when they were applied, as well as the determination of the optimal number of days used to form the filter. Stacking more days' residuals initially improves the overall coordinate precision, up to the optimum case of stacking seven days' residuals, after which coordinate precision drops.

\section{Multi-Antenna Arrays}

The concept of a multiple antenna array has been discussed previously in several investigations with different perspectives. One of the earliest was by Santerre and Beutler (1993), who linked multiple antennae to a GPS receiver with the aim of improving height determination for baselines of few kilometres in length. Forward et al. (2001) developed a 
GPS switched antenna array system, recording data from different stations quasicontinuously for steep wall deformation monitoring at Mount Keith, Western Australia. Their system comprised four switched antennae, in addition to two continuously operating reference stations recording at $1 \mathrm{~Hz}$. 3D deformations of $2 \mathrm{~mm} /$ week were detected. Chen et al. (2000) and Ding et al. (2000) tested a GPS Multi-antenna System (GMS) to monitor deformations such as landslides or unstable slopes. Their results reached centimetre precision, which is near to the typical accuracy achieved using conventional GPS surveying systems.

He et al. (2004a, b) used a more developed prototype of GMS with eight channels, called GMAS (GPS Multi-Antenna System), for monitoring the Xiaolangdi dam on the Yellow River in China. The average precision of baselines over the whole observation period reached around 1-2 mm. The same methodology was applied in He et al. (2005) on the Xiaowan dam on the Lanchang River in Yunnan province, China. This application included 16 monitoring stations connected to two GPS receivers, and two base stations with individual receivers. An overall accuracy of $3 \mathrm{~mm}$ in the horizontal direction and $7 \mathrm{~mm}$ in the vertical direction was achieved. Recently, Ding et al. (2007) used GMAS for monitoring landslides over the Heihe reservoir in Xi'an, China, as well as monitoring roadside slopes. For a three-hour batch solution, an accuracy of $\sim 2 \mathrm{~mm}$ was achieved in all three coordinate components under typical slope site conditions.

The switch device used in the present investigation includes four connections, two for the two antennae, one serial connection to the receiver, and a parallel interface to connect to the PC. Another serial port can also be connected from the receiver to the PC. In addition, the switch box contains a 9-12V DC power socket, a manual antenna override switch, and two indicators to show which antenna is active. All described connections are schematically shown in Figure 1. A Visual Basic program is used to control the connection from each antenna to the receiver. An important role of the program is to synchronize the PC clock with Coordinated Universal Time (UTC) so that the switch time remains synchronized relative to GPS time. This is achieved using the independent Windows utility program Tardis95.

\section{Optimum Antenna Switching Interval}

An important requirement in terms of the switching interval is to achieve the optimum amount of usable data, whilst maintaining the ability to monitor rapid displacements of each antenna. The interval should be long enough not only for the receiver to regain full lock on all 
visible and healthy satellites, but also to obtain enough reliable observations from these satellites to demonstrate consistently accurate solutions. In addition, the interval must be valid for the application of sidereal filtering. To investigate these factors, three stations were chosen on the roof of the Drummond building on the Newcastle University campus (DRMN, DRMS and DRME; Figure 2). Two LEICA GX1200 receivers and three antennae were used. One receiver was connected directly to the antenna at DRMN. The other receiver was connected to the two antennae at DRMS and DRME, via the switch device. Previous multipath analysis in Ragheb (2008), using the code multipath proxy MP1 and MP2 values (Estey and Meertens 1999), showed that DRME suffers from very high multipath for low and medium elevation satellites compared with DRMN and DRMS, as a result of infrastructure close to DRME station.

The first GPS campaign used here included four different 48-hour datasets observed continuously at DRMN and switching between DRMS and DRME at intervals of 1, 2, 5 and 10 minutes. The raw DBX (Direct Branch eXchange) files for both the base and switched receivers were transformed into RINEX format using an automated script based on the TEQC software (Estey and Meertens 1999). A separate RINEX file was extracted for each switched antenna. Processing was then executed with GASP independently for each baseline from fixed site DRMN, using IGS rapid precise orbits (International GNSS Service 2007).

The East, North, Up and 3D discrepancies from the known coordinates of DRME and DRMS were calculated. Figure 3 illustrates the $50^{\text {th }}, 95^{\text {th }}$ and $99^{\text {th }}$ percentile values of these discrepancies. For example, the $50^{\text {th }}$ percentile values indicate that a time interval ranging from 30-60 seconds following switching is required to achieve centimetre accuracy for $50 \%$ of the data, due to the time required to fully lock on to enough satellites with reliable observations. Larger discrepancies generally result from epochs with too few satellites or partial (single-frequency) or miscorrelated carrier phase data, leading to unsuccessful ambiguity resolution. The time to full lock will to some extent depend on the receiver/antenna environment, hardware, switching mechanism, and cables used. For example, the higher-multipath DRME station does not achieve centimetric accuracy with 95\% probability even after $600 \mathrm{~s}$, whereas DRMS reaches this state after around $200 \mathrm{~s}$. The one minute switching interval does not provide a sufficient a number of accurate epochs (perhaps only 15-20 seconds) before switching to the other antenna, and therefore this interval is not considered further. 
In addition, Figure 3 indicates that the 2, 5 and 10 minute switching intervals require almost the same reacquisition time for $50 \%$ probability of centimetric raw coordinate accuracy. From the perspective of rapid deformation monitoring, the shorter 2 minute and 5 minute intervals are the best intervals to use, and the remaining question is which interval is most compatible with sidereal filtering. With the optimum value of the sidereal lag being $86154 \mathrm{~s}$, the 5 minute (300 s) interval cannot be synchronized with this lag value. Even using a range of $\pm 20 \mathrm{~s}$, it is impossible to find matching epochs for filtering after an integer number of switch sessions.

On the other hand, for the 2 minute switching interval (120 s), using a lag value of $86160 \mathrm{~s}$ that is $6 \mathrm{~s}$ away from the optimum lag will provide matching epochs after 718 switch sessions (359 sessions per antenna). Better still, using a switching interval of $119 \mathrm{~s}$ ( $1 \mathrm{~s}$ off the twominute mark) will provide matching epochs after just 362 sessions per antenna with a lag value of $86156 \mathrm{~s}$ (23h 55m 56s). This lag value is only $2 \mathrm{~s}$ away from the optimum lag. Consequently, the modified two minute switch (119 s instead of $120 \mathrm{~s}$ ) is the appropriate interval to use, for two reasons. The first is that it provides an adequate number of accurate epochs per switch session. Secondly, due to its ease of synchronization with the sidereal lag, it allows the use of sidereal filtering to improve coordinate quality. Note that changes in the signal-to-noise ratio (SNR) at the receiver, receiver hardware and configuration, or antenna multipath environment, may affect the reacquisition time and make shorter or longer switching intervals optimal.

\section{Combined Technique Applied to Deformation Monitoring}

In continuation to the results obtained in the previous section, we next tested the ability of the combined sidereal filter and switched antenna array system to resolve measured displacements. For this purpose, measured antenna displacements were implemented using a purpose-built, rigid and adjustable platform (Figure 4). This displacement simulation platform is a tri-axial platform mounted on pillar DRME and fixed with a screw bolt and steel guy-ropes to prevent extraneous movement. Movement on each axis is controlled separately by a knob connected to a precisely threaded spindle. Two antennae are mounted on the platform's flanges, called DRME_A and DRME_B hereafter. These antennae were connected to a receiver via the switch, whilst another antenna on DRMN was connected continuously to a second receiver. Data were collected for 10 days using the same types of receivers and 
antennae, while switching between the DRME_A and DRME_B antennae using the modified “two-minute” switching interval of 119 seconds.

GPS observations were collected every switching interval during the first eight sidereal days without moving the platform, after which various magnitudes of displacements were applied in the horizontal and vertical directions. At first, increasing horizontal displacements were implemented every hour along the platform-centred $X$ axis shown in Figure 4, with increments of 1, 2, 5, 10, 20, 50 and finally $100 \mathrm{~mm}$. The following day the same magnitude displacements were applied, also at hourly intervals, but in the vertical direction (the platform $\mathrm{Z}$ axis). Each implemented displacement was measured using a digital vernier calliper of 0.01 mm accuracy.

The IGS ultra-rapid predicted orbits (International GNSS Service 2007) are used here to obtain satellite coordinates, in order to confirm the ability to monitor structural deformations in near-real time. The first half of each of these 48-hour orbit files is observed, whilst the second half is predicted. New orbit files are released four times per day with a latency of 3 hours from the end of the observed part of the orbits. Accordingly, the next six hours of predicted satellite orbits in each file were extracted, and then concatenated with their counterparts in other files. In this way, satellite orbits are generated which simulate the most accurate predictions available continuously in real time. The GPS data were then processed for both switch antennae, fixing the coordinates of DRMN, after which sidereal filtering was applied using the coordinate residuals from the first eight days of observations.

A tolerance limit of $30 \mathrm{~cm}$ from the long term average coordinate value was adopted for accepting coordinates for sidereal filter generation and later analysis. This acceptance limit is necessary, because in weak satellite geometry, some epochs may pass the AFV threshold and have low formal errors, but are still demonstrably unreliable when compared with the known coordinates, and also to eliminate the bad epochs after switching from one antenna to the other. This acceptance limit provides a balance between monitoring different magnitudes of deformations, and being able to distinguish between good and poor epochs.

Figure 5 shows the unfiltered coordinate time series for DRME_A for East and North while implementing horizontal displacements, and for the Up direction while implementing vertical displacements. DRME_B displays very similar behaviour. During the time span shown, seven different magnitudes of displacements were applied, in addition to about 1.5 hours of data before and after employing these displacements. By applying a 119-second box-car filter 
for the coordinates for each switching interval, a moving average coordinate is overlaid on the coordinate time series, which illustrates more clearly the behaviour of the time series. Note that the $\mathrm{Y}$ axis scale is different among all three directional components due to the varying effect of the applied displacement in each direction (the platform axes are not aligned to true North).

Based on the recommendations of Ragheb et al. (2007a), seven days of coordinate residuals prior to the displacement application were stacked to form the filter, at the optimum sidereal lag that is possible with the adopted switching interval of 86156 seconds. Then, these residuals are subtracted to obtain final filtered coordinates. Figure 6 shows the corresponding coordinate time series after applying sidereal filtering. These coordinates are more compact and homogeneous, as expected. Consequently, we conclude that sidereal filtering is useful to improve coordinate precision, when combined with antenna switching.

Note that some parts of the filtered data were not vastly improved after filtering, which is mainly because of the use of a relatively large acceptance limit $(30 \mathrm{~cm})$ causing some filtered residuals within the 7-day-stack to include epochs that are in error by up to $30 \mathrm{~cm}$. The existence of spikes in the filtered coordinate time series implies either that the unfiltered coordinates were off by a large value which the applied filter could not mitigate, or the filter did not include any value for these epochs. The latter is possible even while stacking seven days of data, as matching epochs occur at the same time relative to each switch session and within the first 30-50 epochs these frequently fail the $30 \mathrm{~cm}$ acceptance criterion.

\section{Results and Discussion}

In order to assess the efficiency of sidereal filtering, an hourly batch average is calculated for each coordinate component and plotted with error bars showing 3 standard deviations. Figure 7 shows the unfiltered and filtered hourly average coordinates in all three directions, while applying horizontal displacements; Figure 8 is formed similarly but for the case of implementing vertical displacements.

After applying sidereal filtering it becomes easier to detect small displacements, as the noise in the data (being mainly multipath) is reduced. A very important aspect seen here is the homogeneity and stability of the coordinates after filtering which is clear from the reduction in the standard deviation, especially in the direction with no effective displacements. In other words, sidereal filtering reduces the multipath error affecting the data and thus any remaining 
steps or jumps in the time series are purely due to the component of the actual displacement in each direction. This demonstrates the ability to detect, at the few-millimetre level, movements in the horizontal and vertical directions which were not apparent before filtering because multipath was causing noise in the time series, as can be seen from the hourly moving average.

Figure 9 shows the true displacements plotted against the observed unfiltered and filtered displacements. In each component, all epochs are plotted within each hour, overlaid by the 119-second session averages and then by the hourly average. The slope of the best fit to the displacements as well as the overall RMS of the residuals to the best fit is given, for each of the three plotted cases. It can be seen that the RMS of the residuals to the best fit decreases as the coordinates are averaged over each session and then over each hour. Additionally, the RMS of the residuals for the filtered coordinates is lower than for the unfiltered coordinates, for all directional components and cases. Figure 9 indicates also that sidereal filtering in most cases not only confines the scatter of the displacements around the average observed value (improves precision), but also brings them closer to the true value, (improves accuracy).

Using the combined technique of single-epoch switched antenna array GPS and sidereal filtering, one can reach an overall RMS for the residuals in the horizontal plane of $4.5 \mathrm{~mm}$ when considering all individual epochs, $2.7 \mathrm{~mm}$ for the "two-minute" switch session average and $0.6 \mathrm{~mm}$ for the hourly average, with an average ratio of 0.99 between the true and observed horizontal displacements. These compare with, for example, precisions of 1-3 mm achieved over much longer sessions with the GMAS technique (Ding et al., 2007). On the other hand, the RMS for the Up coordinates reaches $7.5 \mathrm{~mm}$ for individual epochs, $5.5 \mathrm{~mm}$ for session averages and $0.9 \mathrm{~mm}$ for hourly averages, again with a mean ratio of 0.99 between true and observed displacements.

\section{Conclusions}

The multi-antenna switch array system provides semi-continuous GPS observations in a costeffective manner. Unfortunately, switching between antennae will result in some poor epochs of data at the start of each session until lock is regained on sufficient healthy satellites for reliable positioning. The number of these transitional epochs was discovered to be about 30 50 seconds regardless of the switching interval, for the tested hardware and environment. Consequently, the modified "two-minute" switching interval (119 seconds) is the most appropriate session interval to use, producing a sufficient number of good epochs per 
switching interval, reliable precision, and in addition being short and easily synchronized with the most suitable lag (86156 seconds) for sidereal filter application.

Using switched antenna GPS data, which for the given situation resulted typically in the loss of about 40 seconds of usable data at the start of each switching interval, displacements were detected with an acceptable precision especially after sidereal filtering. Use of a shorter GPS recording interval (e.g. 10-20 Hz) would not in itself decrease the time of data loss, but would result in a greater number of usable epochs. Accordingly, the adopted methodology accomplished the required target of monitoring deformations using a quick, cost-effective and precise GPS technique.

Applying sidereal filtering to periodic GPS data reduces sidereally repeating errors, mainly multipath, which makes it easier to differentiate between noise and genuine deformation. The combined method of single epoch, sidereal filtering and multi-antenna array was successful in detecting horizontal and vertical displacements in simulated near-real time with reduced errors reaching the few-millimetre level despite a high multipath environment. The instantaneous coordinate precision improved vastly, reaching better than $5 \mathrm{~mm}$ and $8 \mathrm{~mm}$ in the horizontal and vertical directions respectively.

\section{References}

Agnew, D.C. and Larson, K.M. (2007). "Finding the Repeat Times of the GPS Constellation." GPS Solutions, 11(1), 71-76.

Al-Haifi, Y. (1996). "Short Range GPS Single Epoch Ambiguity Resolution." PhD Thesis, Department of Surveying, University of Newcastle upon Tyne, Newcastle upon Tyne, UK.

Axelrad, P., Comp, C.J. and MacDoran, P.F. (1996). "SNR-based Multipath Error Correction for GPS Differential Phase." IEEE Trans. Aerosp. and Electr. Syst., 32(2), 650-660.

Bock, Y., De Jonge, P.J., Honcik, D., Bevis, M., Bock, L. and Wilson, S. (2001). "Epoch-ByEpoch Positioning Applied to Dam Deformation Monitoring at Diamond Valley Lake, Southern California." Proc. $10^{\text {th }}$ FIG Int. Symp. on Deformation Measurements, Orange, CA, USA.

Bock, Y., Nikolaidis, R.M., De Jonge, P.J. and Bevis, M. (2000). "Instantaneous Geodetic Positioning at Medium Distances with the Global Positioning System." J. Geophys. Res., 105(B12), 28223-28253. 
Chen, Y.Q., Ding, X.L., Huang, D.F. and Zhu, J.J. (2000). "A Multi-antenna GPS System for Local Area Deformation Monitoring." Earth Planet. Space, 52(10), 873-876.

Choi, K., Bilich, A., Larson, K.M. and Axelrad, P. (2004). "Modified Sidereal Filtering: Implications for High-rate GPS Positioning." Geophys. Res Lett., 31(L22608), 1-4.

Corbett, S. (1994). "GPS Single Epoch Ambiguity Resolution for Airborne Positioning and Orientation." PhD Thesis, Department of Surveying, University of Newcastle upon Tyne, Newcastle upon Tyne, UK.

Counselman, C.C. and Gourevitch, S.A. (1981). "Miniature Interferometer Terminals for Earth Surveying: Ambiguity and Multipath with Global Positioning System." IEEE Trans. Geosci. Remote Sens., G-19(4), 244-252.

De Jonge, P.J., Bock, Y. and Bevis, M. (2000). "Epoch-by-Epoch Positioning and Navigation." Geodetics Inc., La Jolla, CA, USA.

Ding, X.L., Chen, Y.Q., Huang, D., Zhu, J., Tsakiri, M. and Stewart, M. (2000). "Slope Monitoring using GPS: A Multi-Antenna Approach." GPS World, 11(3), 52-55.

Ding, X.L., Dai, W.J., Yang, W.T., Zhou, X.W., Lam, J., Zhang, Q. and Wang, L. (2007). "Application of Multi-Antenna GPS Technology in Monitoring Stability of Slopes." Strategic Integration of Surveying Services, Proc. FIG Working Week 2007, Hong Kong, China.

Estey, L.H. and Meertens, C.M. (1999). "TEQC: The Multi-Purpose Toolkit for GPS/GLONASS Data." GPS Solutions, 3(1), 42-49.

Forward, T., Stewart, M., Penna, N. and Tsakiri, M. (2001). "Steep Wall Monitoring using Switched Antenna Arrays and Permanent GPS Networks." Proc. $10^{\text {th }}$ FIG Int. Symp. on Deformation Measurements, Orange, CA, USA.

Genrich, J.F. and Bock, Y. (1992). "Rapid Resolution of Crustal Motion at Short Ranges with the Global Positioning System." J. Geophys. Res., 97(B3), 3261-3269.

Gunasingam, R. (2003). "Single Epoch Ambiguity Resolution using Low-Cost GPS Receivers." MPhil Thesis, School of Civil Engineering and Geosciences, University of Newcastle upon Tyne, Newcastle upon Tyne, UK.

He, X.F., Sang, W., Chen, Y.Q. and Ding, X.L. (2005). "Steep Slope Monitoring: GPS Multiple Antenna System at Xiaowan Dam." GPS World, 16(11), 20-22, 24-25.

He, X.F., Guang, Y., Ding, X.L. and Chen, Y.Q. (2004a). "Application and Evaluation of a GPS Multi-antenna System for Dam Deformation Monitoring." Earth Planet. Space, 56(11), 1035-1039. 
He, X.F., Guang, Y., Ding, X.L. and Chen, Y.Q. (2004b). "Development of GPS Multiantenna Switch for Deformation Monitoring." Proc. Int. Symp. on GNSS/GPS, Sydney, Australia.

International GNSS Service (2007). "IGS Product Availability" Data and Products, $<$ http://igscb.jpl.nasa.gov/components/prods_cb.html> (Sep. 8, 2007).

Larson, K.M., Bilich, A. and Axelrad, P. (2007). "Improving the precision of high-rate GPS." J. Geophys. Res., 112(B05422), 1-11.

Lau, L. and Mok, E. (1999). "Improvement of GPS Relative Positioning Accuracy by using SNR." J. Surv. Eng., ASCE, 125(4), 185-202.

Leick, A. (2004). "GPS Satellite Surveying." Second Edition, John Wiley \& Sons Inc, NY, USA.

Nikolaidis, R.M., Bock, Y., De Jonge, P.J., Shearer, P., Agnew, D.C. and Van Domselaar, M. (2001). "Seismic Wave Observations with the Global Positioning System." J. Geophys. Res., 106(B10), 21897-21916.

Ogaja, C., Rizos, C., Wang, J. and Brownjohn, J. (2001). "Toward the Implementation of Online Structural Monitoring using RTK GPS and Analysis of Results using the Wavelet Transform." Proc. 10 ${ }^{\text {th }}$ Int. Fed. Surv. (FIG), Working Group 6, Deformation Measurements and Analysis, Orange, CA, USA, 284-293.

Ragheb, A.E. (2008). "Filtered and Semi-Continuous Single Epoch GPS for Deformation Monitoring." PhD Thesis, School of Civil Engineering and Geosciences, Newcastle University, Newcastle upon Tyne, UK.

Ragheb, A.E., Clarke, P.J. and Edwards, S.J. (2007a). "Coordinate-space and Observationspace Filtering Methods for Sidereally Repeating Errors in GPS: Performance and Filter Lifetime." Proc. ION NTM 2007, Catamaran Resort Hotel, San Diego, CA, USA, 480485.

Ragheb, A.E., Clarke, P.J. and Edwards, S.J. (2007b). "GPS Sidereal Filtering: Coordinateand Carrier-phase-level Strategies." J. Geodesy, Berlin, 81(5), 325-335.

Remondi, B. (1984). "Using the Global Positioning System (GPS) Phase Observable for Relative Geodesy: Modelling, Processing and Results." PhD Thesis, Cent. Sp. Res., University of Texas, Austin, TX, USA.

Roberts, G.W., Meng, X. and Dodson, A.H. (2001). "Data Processing and Multipath Mitigation for GPS/Accelerometer Based Hybrid Structural Deflection Monitoring 
System." Proc. $14^{\text {th }}$ ITM of the Satellite Division of the Institute of Navigation (ION GPS-2001), Salt Palace Convention Centre, Salt Lake City, UT, USA, 473-508.

Roberts, G.W., Meng, X. and Dodson, A.H. (2004). "Integrating a Global Positioning System and Accelerometers to Monitor the Deflection of Bridges." J. Surv. Eng., 130(2). 65-72. Rutledge, D., Gnipp, J. and Kramer, J. (2001). "Advances in Real-Time GPS Deformation Monitoring for Landslides, Volcanoes and Structures." Proc. $10^{\text {th }}$ FIG Int. Symp. on Deformation Measurements, Orange, CA, USA.

Santerre, R. and Beutler, G. (1993). "A Proposed GPS Method with Multi-Antennae and Single Receiver." J. Geodesy, Berlin, 67(4), 210-223. 


\section{Figures}

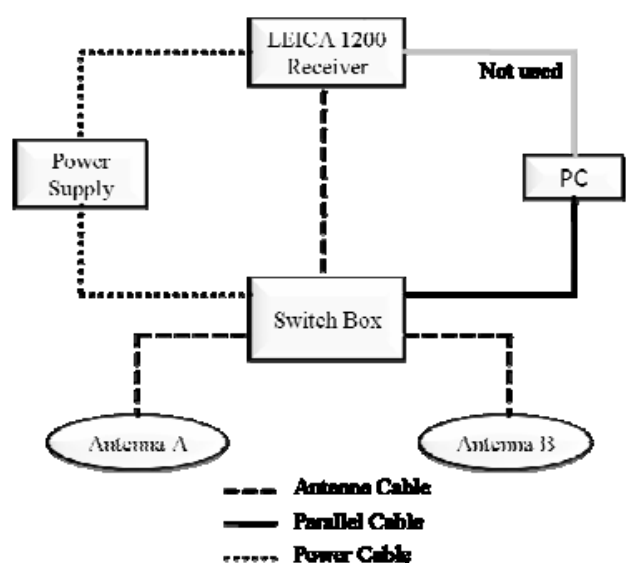

Figure 1. Schematic diagram of antenna array connections.

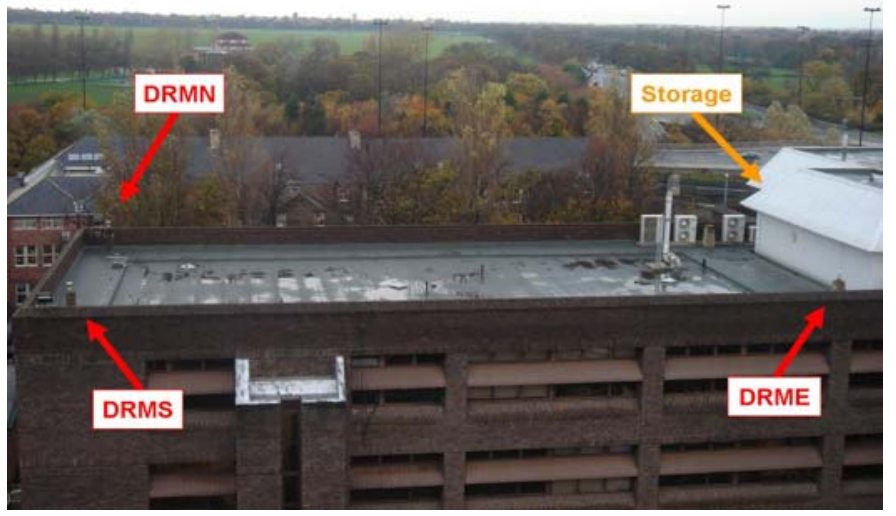

Figure 2. Relative location of the three Drummond building stations.

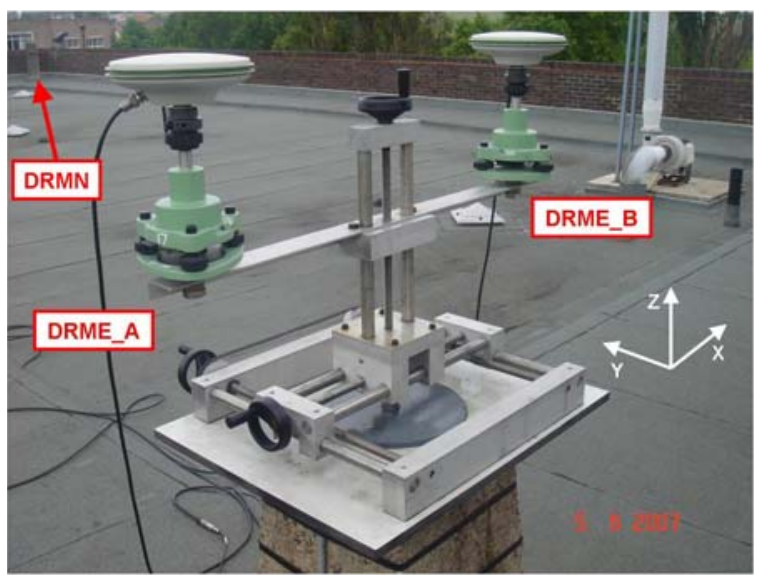

Figure 4. DRME A and B antennae mounted on the displacement platform. 

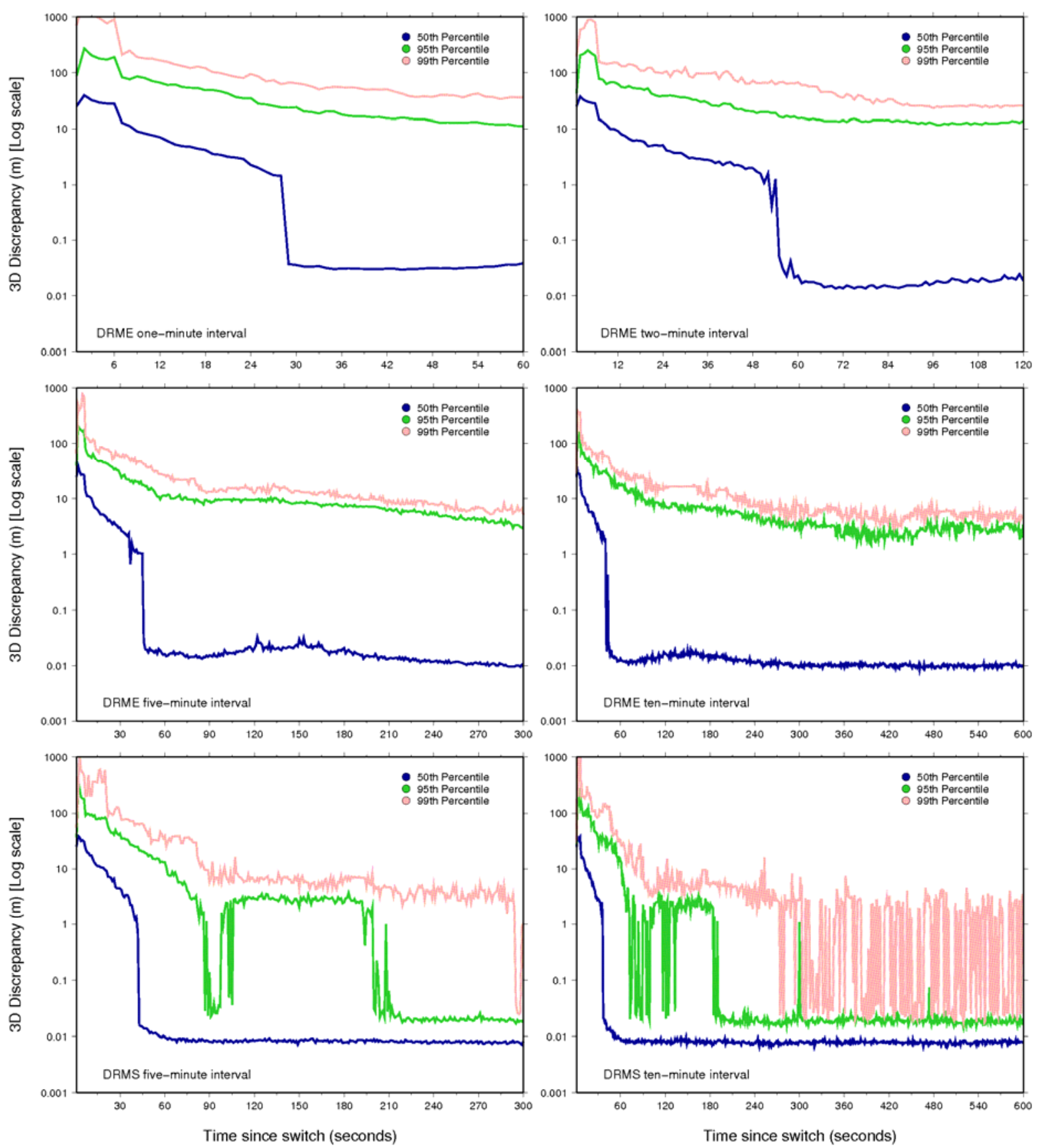

Figure 3. 3D coordinate discrepancy percentiles for all switching intervals: (top left) DRME one-minute interval, (top right) DRME two-minute interval, (mid left) DRME five-minute interval, (mid right) DRME tenminute interval, (bottom left) DRMS five-minute interval, (bottom right) DRMS ten minute-interval. The figures for the DRMS one-minute and two-minute intervals closely resemble those at DRME so are not shown. 

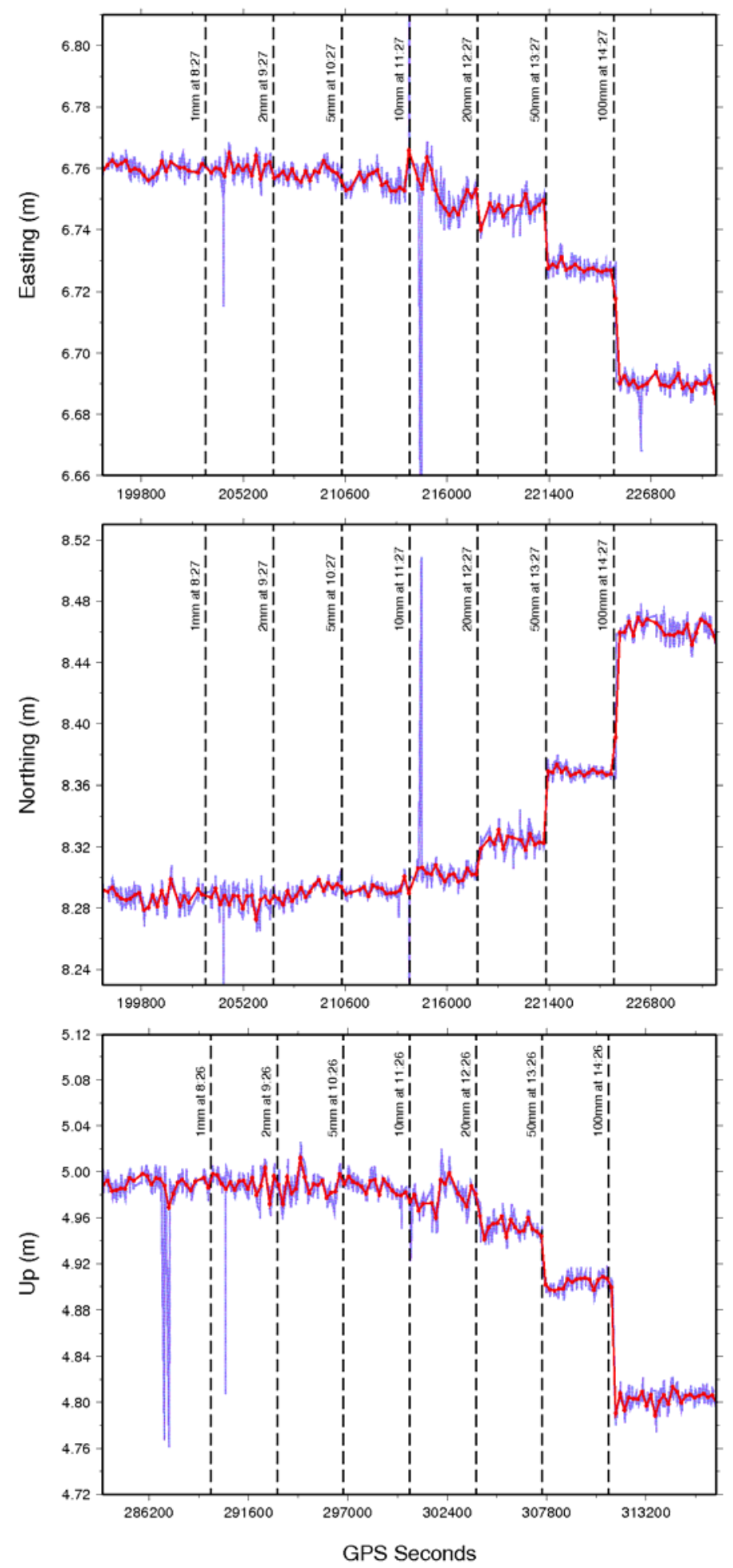

Figure 5. DRME_A unfiltered coordinate time series (GPS week 1445, relative to false origin). East and North while implementing horizontal displacements; Up while implementing vertical displacements. Black dashed lines indicate times and magnitudes of these displacements. Smoothed line shows a 119 s box-car filter. 

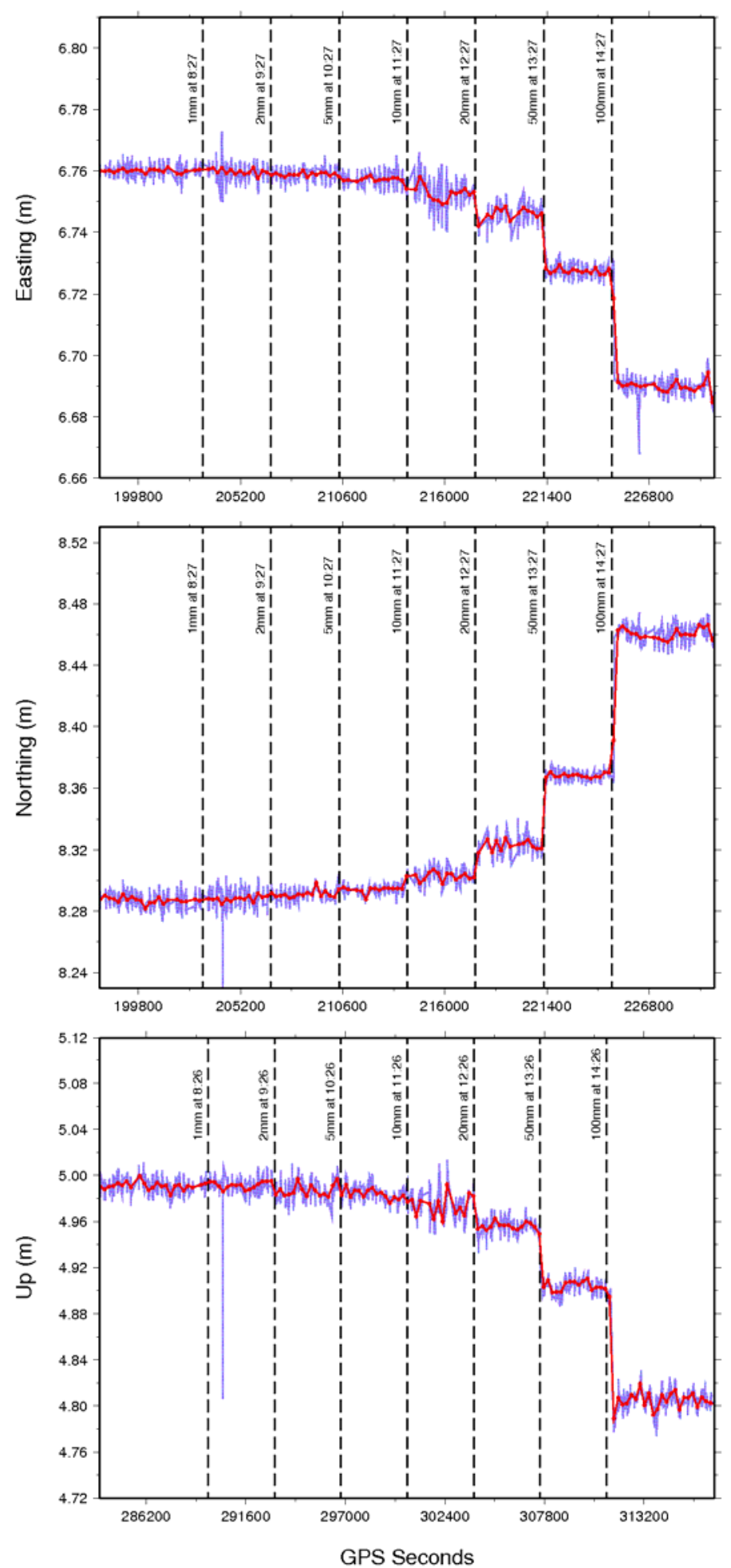

Figure 6 DRME_A filtered coordinate time series. Line attributes, configuration, coordinates origin and GPS week as before. 


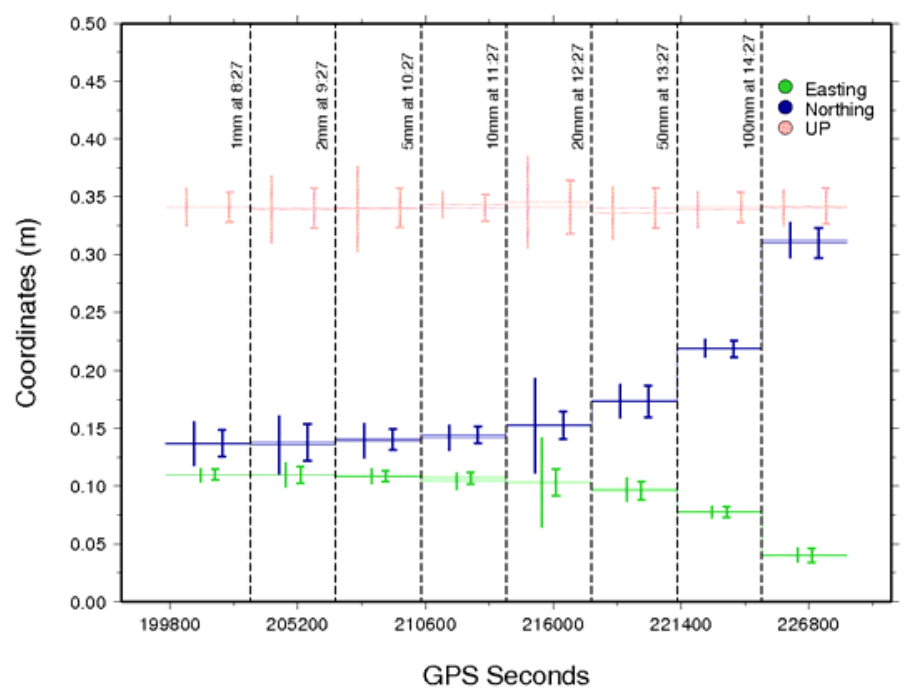

Figure 7. DRME_A unfiltered and filtered average hourly coordinates (GPS week 1445, relative to false origin) whilst applying horizontal displacements. Solid lines and right error bars for filtered coordinates; dotted lines and left error bars for unfiltered coordinates. Error bars represent 3 standard deviations.

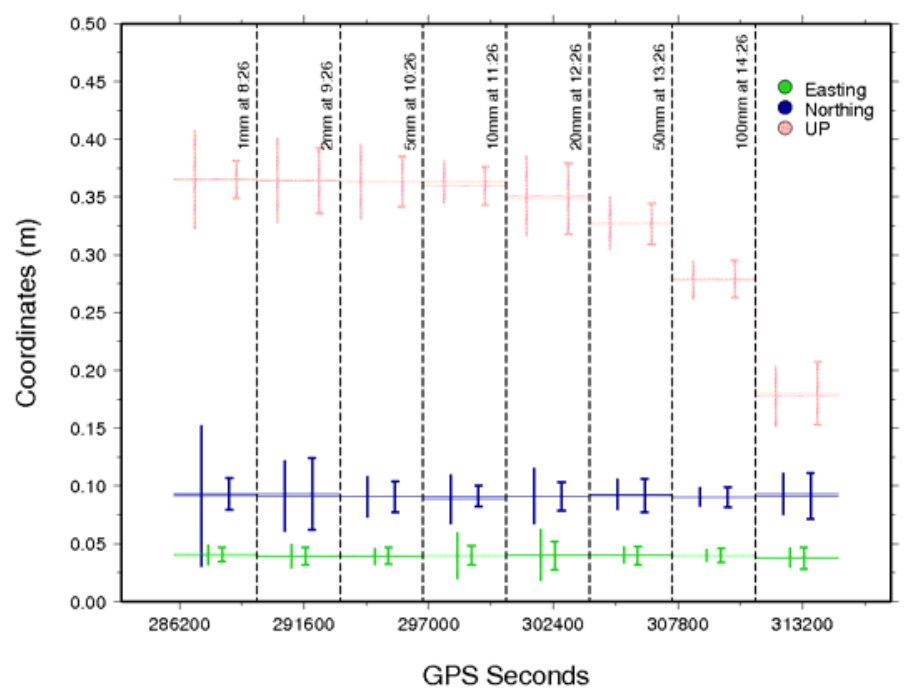

Figure 8. DRME_A unfiltered and filtered average hourly coordinates whilst applying vertical displacements. Line attributes, error bars, coordinates origin and GPS week as before. 
Unfiltered
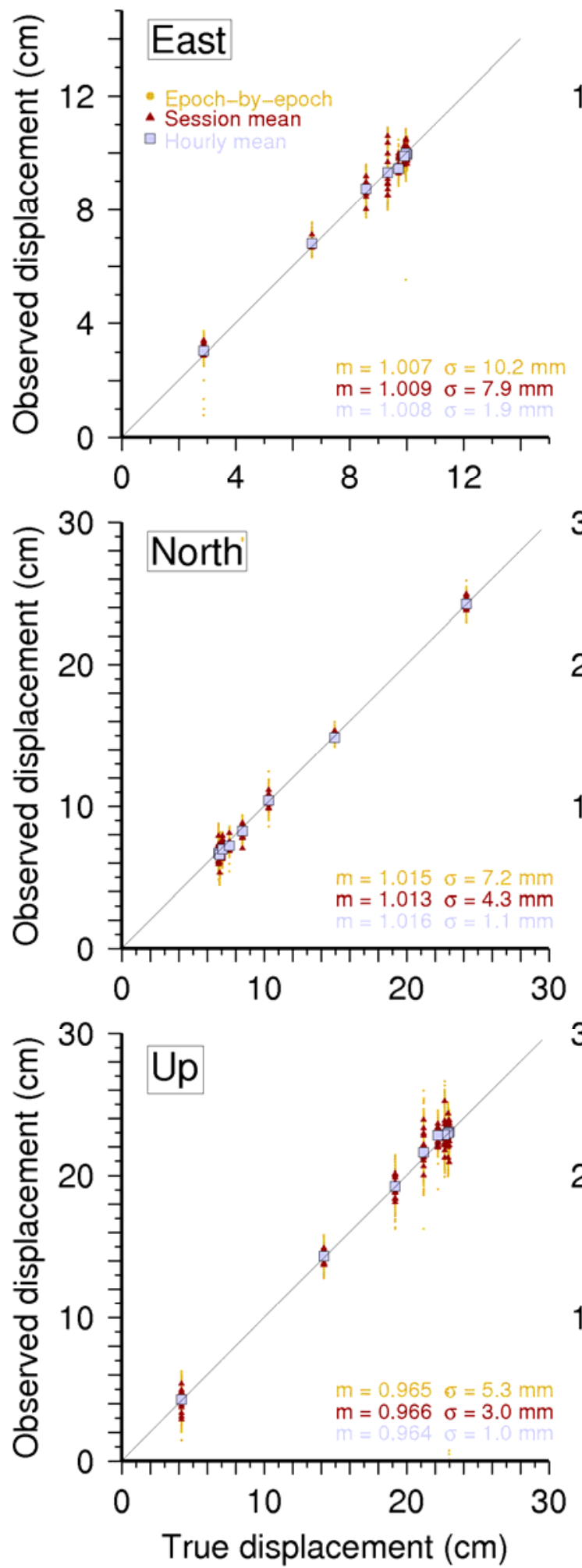

Filtered
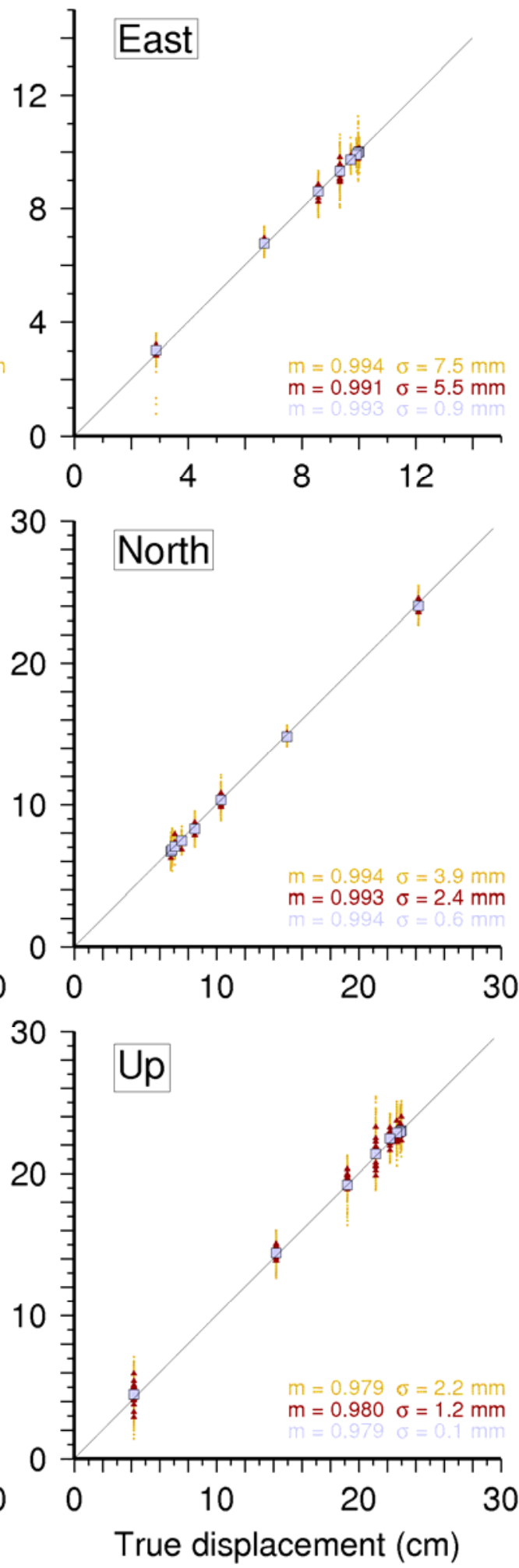

Figure 9. DRME_A observed (unfiltered and filtered) coordinates versus true displacements. Black line indicates ideal relation. 